BULL. AUSTRAL. MATH. SOC.

$28 A 25$

VOL. 31 (1985), 389-411.

\title{
TRAJECTORIES OF SET VALUED INTEGRALS
}

\author{
Nikolaos S. Papageorgiou
}

The purpose of this paper is to study the trajectory multifunction $\Phi(\cdot)$ determined by the indefinite set valued integral of a measurable Banach space valued multifunction $F(\cdot)$, that is for all $t \in[0, T], \Phi(t)=\int_{0}^{t} F(s) d s$, where the set valued integral is interpreted in the sense of Aumann. We study the topological and algebraic properties of $S_{\Phi}$ equaling the set of selectors of $\Phi(\cdot)$ whose primitive is an integrable selector of $F(\cdot)$. We also determine several useful properties that $\Phi(\bullet)$ possesses and finally we present some convergence and stability results using the Kuratowski-Mosco convergence of sets.

\section{Introduction}

Consider the multifunction $F:[0, T]+2^{X}$, where $X$ is a Banach space. We are interested in studying the properties of the set valued integrals defined by $\Phi(t)=\int_{0}^{t} F(s) d s, t \in[0, T]$. The importance of this multifunction comes from the fact that it is essentially (that is after an appropriate substitution) the attainable set of a linear control system. More specifically we have a linear control system determined by the following evolution equation

Received 6 November 1984. Supported by N.S.F. - Grant DMS-8403135.

Copyright Clearance Centre, Inc. Serial-fee code: 0004-9727/85 $\$ A 2.00+0.00$. 


$$
\dot{x}=A(t) x+g(t, u)
$$

where $A(\cdot)$ is a densely defined, closed linear operator which generates a strongly continuous semigroup and for every $u \in U$ equalling another Banach space, $f(\cdot, u) \in L_{X}^{1}(T) \quad(T=[0, T])$. The set of all admissible controls is the set of selectors of a multifunction (often called the "control multifunction") $\Gamma: T \rightarrow 2^{U}$. Denote by $x=x(\cdot, u)$ the solution of $(*)$ corresponding to an admissible control function $u(\cdot)$ and fixed initial condition. The set

$$
A(t)=\{x(t, u): u=\text { admissible control function }\}
$$

is called the attainable set and is of major importance in the study of control systems. This set is up to a linear transformation and translation the "integral" of the multifunction $F(\cdot)$ defined by

$$
F(\cdot)=\{x \in X: x=R(t, 0) f(t, u), u \in \Gamma(t)\}
$$

where $R(\cdot)$ is the semigroup of operators generated by $A(\cdot)$ (see [8]).

Also in control theory, as well as in mathematical economics we often encounter generalized differential equations (differential inclusions) of the form $\dot{x}(t) \in F(t, x(t)), x(0)=x_{0}$. Such equations are also called contingent equations and set valued integration is a basic tool in their study (see Hermes [14], [15]).

Such applications motivated the present work.

Briefly the organization of the paper is as follows. In Section 2 we establish our terminology and notational conventions and we recall some basic facts from the theory of multifunctions. In Section 3 we study the set of selectors of the trajectory multifunction $t \rightarrow \Phi(t)=\int_{0}^{t} F(s) d s$. In Section 4 we examine the properties of $\Phi(\cdot)$. Finally in Section 5 we present some convergence theorems using the Kuratowski-Mosco convergence of sets and we also establish a stability property of $\Phi(\cdot)$.

\section{Preliminaries}

In this section we fix our terminology and notation and we recall some basic facts about multifunctions. For more details we refer to Castaing 
and Valadier [6], Himmelberg [18], Himmelberg et al [19], Hildebrandt [17] and Rockafellar [23], [24].

So let $F: \Omega \rightarrow 2^{X}$ be a multifunction (set valued function) from the space $\Omega$, to the power set of the space $X$. The graph of $F(\cdot)$ is the set $\operatorname{Gr} F=\{(\omega, x) \in \Omega \times X: x \in F(\omega)\}$. Also if $V \subseteq X$ the weak inverse image of $V$ by $F(\cdot)$ is the set $F^{-}(V)=\{\omega \in \Omega: F(\omega) \cap V \neq \emptyset\}$. When $X$ is a topological space we will use the following notation:

$$
\begin{aligned}
& P_{f(c)}(X)=\{A \subseteq X: \text { nonempty, closed, (convex) }\} ; \\
& P_{k(c)}(X)=\{A \subseteq X: \text { nonempty, compact, (convex) }\} .
\end{aligned}
$$

A $w$ in from of $f$ (respectively $k$ ) will mean that the closedness (respectively compactness) is with respect to the weak topology $w\left(X, X^{*}\right)$. The next theorem summarizes the major results about the measurability of closed valued multifunctions.

THEOREM 2.1. Let $(\Omega, \Sigma)$ be a measurable space and $X$ a separable metric space. Let $F: \Omega \rightarrow P_{f}(X)$ be a multifunction. Consider the following statements:

(1) $F^{-}(B) \in \Sigma$ for every $B \in B(X)$ equalling the Borel o-field of $X$;

(2) $F^{-}(C) \in \Sigma$ for every $C \subseteq X$ closed subset of $X$;

(3) $F^{-}(U) \in \Sigma$ for every $U \subseteq X$ open subset of $X$;

(4) $\omega \rightarrow d(x, F(\omega))$ is a measurable function for alz $x \in X$;

(5) there exists a sequence of measurable selectors $\left\{f_{n}(\cdot)\right\}_{n \geq 1}$ of $F(\cdot)$ such that $\operatorname{cl}\left\{f_{n}(\omega)\right\}=F(\omega)$ (Castaing's representation of $F(\cdot)$ );

(6) $\operatorname{Gr} F \in \sum \times B(X)$.

Then we have the following results:

(i) (1) $\Rightarrow$ (2) $\Rightarrow$ (3) $\Leftrightarrow$ (4) $\Rightarrow$ (6);

(ii) if $X$ is Polish (that is, is in addition complete) then (3) $\Longleftrightarrow(5)$;

(iii) if $X$ is Polish and there is a complete $\sigma$-finite measure 
on $\Sigma$ then $(1)+(6)$ are all equivalent.

Following Himmelberg [18] we will say that $F: \Omega \rightarrow P_{f}(X)$ satisfying (I) (respectively (2), (3)) is Borel (respectively strong, weakly) measurable.

Suppose now that $X$ is a separable Banach space. For any multifunction $F: \Omega \rightarrow 2^{X}$ we can now define the set

$$
S_{F}^{I}=\left\{f \in L_{X}^{l}(\Omega): f(\omega) \in F(\omega), \mu \text { almost everywhere }\right\},
$$

that is, $S_{F}^{l}$ contains all Bochner integrable selectors of $F(\cdot)$. Clearly $S_{F}^{l}$ may be empty. If it is nonempty and $F(\cdot)$ is closed valued then it is easy to check that it is a closed subset of the Lebesgue-Bochner space $L_{X}^{1}(\Omega)$. Using the set we can now define an integral for $F(\cdot)$. This was first introduced by Aumann [2] and is the natural generalization of the single valued integral and of the Minkowski sum of sets. So we have $\int_{\Omega} F(\omega) d \mu(\omega)=\left\{\int_{\Omega} f(\omega) d \mu(\omega): f \in S_{F}^{l}\right\}$ where the integral of $f(\cdot)$ is in the sense of Bochner. If $|F|(\omega)=\sup _{x \in F(\omega)}\|x\| \in L^{1}$ then we say that $F(\cdot)$ is integrably bounded and in that case $S_{F}^{l} \neq \varnothing$, so that $\int_{\Omega} F(\omega) d \mu(\omega) \neq \emptyset$

Finally we would like to introduce a mode of convergence of sets that is more general than the usual convergence in the Hausdorff metric and which will be our major tool in Section 5 .

So let $X$ be a Banach space and let $\left\{A_{n}\right\}_{n \geq 1} \subseteq P_{f}(X)$. Let $\tau$ be a topology on $X$. We say that $A_{n}$ T-converges to $A$ in the KuratowskiMosco sense $\left(A_{n} \stackrel{\tau K-M}{\longrightarrow} A\right)$ if and only if $\tau-\underset{n}{-\frac{\lim }{n}} A_{n} \subseteq A \subseteq \tau-\frac{\lim }{n \rightarrow \infty} A_{n}$ where $\tau-\frac{\lim }{n} A_{n}=\left\{x \in X: x_{n} \stackrel{\tau}{\longrightarrow} x, x_{n} \in A_{n}, n \geq 1\right\}$ and 
$\tau-\varlimsup_{n \rightarrow \infty} A_{n}=\left\{x \in X: x_{n_{k}} \stackrel{\tau}{\longrightarrow} x, x_{n_{k}} \in A_{n_{k}}, k \geq 1\right\}$. Since we always have that $\tau-\frac{\lim }{n \rightarrow \infty} A_{n} \subseteq \tau-\overline{\lim } A_{n}$ we deduce that $A_{n} \stackrel{\tau K-M}{\longrightarrow} A$ if and only if $\tau-\frac{\lim }{n \rightarrow \infty} A_{n}=A=\tau-\lim _{n \rightarrow \infty} A_{n}$. When $s-\frac{\lim }{n \rightarrow \infty} A_{n}=A=w-\overline{\lim } A_{n}$ where $w=w\left(X, X^{*}\right)$ and $s$ equals the strong (norm) topology on $X$ we say that $A_{n}$ converges to $A$ in the Kuratowski-Mosco sense and we write $A_{n} \stackrel{K-M}{\longrightarrow} A$ as $n \rightarrow \infty$. Using this concept of set convergence we can define a new type of convergence of functions, in general disjoint from the pointwise convergence. So if $\left\{f_{n}, f\right\} \subseteq \bar{R}^{X}$ are proper, closed (that is, lower semi-continuous) functions, then we say that $f_{n} \rightarrow f$ if and only if epi $f_{n} \stackrel{K-M}{\longrightarrow}$ epi $f$ as $n \rightarrow \infty$. For more details we refer to Mosco [21] and Salinetti and Wets [25] and [26].

Throughout this work $T$ wil be the interval $[0, T], \Sigma$ the $\sigma$-field of all Lebesgue subsets of $T$ and $d s$ the Lebesgue measure on $\Sigma$. Also $X$ will be in general a separable Banach space. Additional hypothesis will be introduced as needed. Finally by $\sigma_{A}(\cdot)$ we will denote the support function of $A \subseteq X$, that is, $\sigma_{A}\left(x^{*}\right)=\sup _{x \in A}\left(x^{*}, x\right)$, $x^{*} \in X^{*}$

Our work on the one hand generalizes significantly previous works by Artstein [1], Bridgland [4], [5] and Hermes [14] and on the other hand contains several new results that provide a better understanding of the evolution of an indefinite set valued integral. We believe strongly that researchers from various areas of applied mathematics, such as optimization, optimal control, and mathematical economics, will find here several interesting theoretical results.

\section{The set of selectors of the trajectory multifunction}

Differential inclusions play an important role in optimal control theory. The crucial point in the study of such equations is the compactness of the set of trajectories, from which we can deduce easily the existence of optimal controls. Our first result establishes this desired 
compactness property of the set of trajectories.

THEOREM 3.1. If $F: T \rightarrow P_{w k c}(X)$ is integrably bounded and

$$
\Phi(t)=\int_{0}^{t} F(s) d s, \quad t \in T,
$$

then $\Phi(\cdot)$ is measurable and the set $S_{\Phi}$ of selectors of $\Phi(\cdot)$ which are the indefinite integral of an element in $S_{F}^{1}$ is a compact subset of $C\left(T ; X_{w}\right) \quad\left(X_{w}=X\right.$ with the weak topology $)$.

Proof. If $\varphi(\cdot) \in S_{\Phi}$ then $\varphi(t)=\int_{0}^{t} f(s) d s$ for some $f \in S_{F}^{I}$ and so $\varphi(\cdot)$ is absolutely continuous.

First we will show that $S_{F}^{1}\left(T^{\prime}\right)$ is a w-compact subset of $L_{X}^{1}\left(T^{\prime}\right)$ where $T^{\prime}$ is any closed subinterval of $T$. To prove this we will proceed as follows. Let $g(\cdot) \in\left[L_{X}^{1}\left(T^{\prime}\right)\right]^{*}=L_{X_{W^{*}}^{*}}\left(T^{\prime}\right)$. Then we have

$$
\sup _{f \in S_{F}^{l}\left(T^{\prime}\right)}\langle g, f\rangle=\sup _{f \in S_{F}^{l}\left(T^{\prime}\right)} \int_{T^{\prime}}(g(s), f(s)) d s .
$$

From Theorem 2.1 of $\mathrm{Hiai}$ and Umegaki [16] we have that

$$
\sup _{f \in S_{F}^{\perp}} \int_{T^{\prime}}(g(s), f(s)) d s=\int_{T^{\prime}} \sup _{x \in F(s)}(g(s), s) d s .
$$

Consider $L(s)=\left\{\hat{x} \in F(s):(g(s), \hat{x})=\sup _{x \in F(s)}(g(s), x)\right\}$. Since $F(\cdot)$ is $w$-compact valued $L(s) \neq \varnothing$ for all $s \in T^{\prime}$ and clearly those sets are closed. Let $m(s)=\sup _{x \in F(s)}(g(s), x)$. A straightforward application of Castaing's representation shows that $m(\cdot)$ is measurable. If we set $\psi(s, \hat{x})=(g(s), \hat{x})-m(s)$ then $\psi(\cdot, \cdot)$ is a Caratheodory function and so is jointly measurable on $T^{\prime} \times X$. Thus $\operatorname{Gr} L \in \Sigma_{T^{\prime}} \times B(X)$. Applying Aumann's selection theorem we can find $\hat{x}: T^{\prime} \rightarrow X$ measurable such that $\hat{x}(s) \in L(s)$ for all $s \in T^{\prime}$. Clearly $\hat{x}(\cdot) \in S_{E}^{1}\left(T^{\prime}\right)$. Hence 
$\sup _{f \in S_{F}^{l}\left(T^{\prime}\right)}\langle g, f\rangle=\langle g, \hat{x}\rangle$. Since $g \in L_{\chi_{W^{*}}^{*}}^{\infty}\left(T^{\prime}\right)$ was arbitrary invoking James theorem (see floret [13]) we deduce that $S_{F}^{l}\left(T^{\prime}\right)$ is a w-compact, convex subset of $L_{X}^{1}\left(T^{\prime}\right)$. Hence $\Phi(t)=\int_{0}^{t} F(s) d s \in P_{w k c}(X)$. Next observe that for all $x^{*} \in X^{*}$ we have $\begin{aligned} \sigma_{\Phi(t)}\left(x^{*}\right) & \\ = & \sup _{z \in \Phi(t)}\left(x^{*}, z\right)=\sup _{z \in \int_{0}^{t} F(s)}\left(x^{*}, z\right)=\sup _{f \in S_{F}^{l}\left(T^{\prime}\right)}\left(x^{*}, \int_{0}^{t} f(s) d s\right) \\ = & \sup _{f \in S_{F}^{l}\left(T^{\prime}\right)} \int_{0}^{t}\left(x^{*}, f(s)\right) d s=\int_{0}^{t} \sup _{x \in F(s)}\left(x^{*}, x\right) d s=\int_{0}^{t} \sigma_{F(s)}\left(x^{*}\right) d s .\end{aligned}$

So $t \rightarrow \sigma_{\Phi(t)}\left(x^{*}\right)$ is absolutely continuous for all $x^{*} \in X^{*}$. Then Theorem III-35 of Castaing and Valadier [6] tells us that $t \rightarrow \Phi(t)$ is measurable. Clearly $S_{\Phi}$ is an equicontinuous, bounded set and $\{\varphi(t)\}_{\varphi \in S_{\Phi}} \in P_{w k e}(X)$. Applying the Arzela-Ascoli theorem we deduce that $S_{\Phi}$ is relatively compact in $C\left(T ; X_{w}\right)$. We need to show that it is closed. For that purpose consider a net $\varphi_{a}$ such that $\varphi_{a} \stackrel{C\left(T ; X_{w}\right)}{\longrightarrow} \varphi \Rightarrow \int_{0}^{t} f_{a}(s) d s \stackrel{w}{\longrightarrow} \varphi(t)$ where $\left\{f_{a}\right\} \subseteq s_{F}^{l}$. From the first part of the proof we know that $S_{F}^{l}$ is $w$-compact in $L_{X}^{1}(T)$. So let $f_{b}$ be a convergent subnet. We have

$$
f_{b} \stackrel{w-L_{X}^{1}(T)}{\longrightarrow} f \in s_{E}^{\perp} \Rightarrow \int_{0}^{t} f_{b}(s) d s \stackrel{w}{\longrightarrow} \int_{0}^{t} f(s) d s \Rightarrow \varphi(t)=\int_{0}^{t} f(s) d s
$$

for all $t \in T$.

Now let $X$ have the Radon-Nikodym property and consider the space of normalized absolutely continuous functions (that is $f(0)=0$ ). We know that any such function is differentiable almost everywhere. Consider the 
subspace $N A C^{1}(T ; X)$ which consists of those functions with $L_{X}^{l}(T)$ derivatives. This becomes a Banach space when endowed with the norm $\|f\|_{N A C^{1}}=\int_{T}\|\dot{f}(s)\| d s$. In that case it is easy to see that $\operatorname{HAC}^{\perp}(T ; X)=L_{X}^{I}(T)$. The first part of the proof of Theorem 3.1 gives us the following interesting corollary.

COROLLARY. $S_{\Phi}$ is a w-compact subset of $N A C^{l}(T ; X)$.

When $E(\cdot)$ is not convex and we convexify it essentially what we do is close topologically the trajectories, as the next result shows.

PROPOSITION 3.1. If $F: T \rightarrow P_{w k}(X)$ is integrably bounded then cl $\Phi(t)=\int_{0}^{t} \overline{\operatorname{conv}} F(s) d s$ and $S_{c l \Phi}=\overline{\operatorname{conv}} S_{\Phi}$ the closure taken in $L_{X}^{1}(T)$

Proof. Corollary 4.3 of [16] tells us that

$$
\mathrm{cl} \int_{0}^{t} \overline{\operatorname{conv}} F(s) d s=c l \int_{0}^{t} F(s) d s \text {. }
$$

Also from the Krein-Smulian Theorem ([10], p. 51) we know that $\overline{\operatorname{conv}} F(s) \in P_{w k c}(X)$ for all $s \in T$. So $\int_{0}^{t} \overline{\operatorname{conv}} F(s) d s$ is closed implies $\operatorname{cl} \Phi(t)=\int_{0}^{t} \overline{\operatorname{conv}} F(s) d s$. Finally recall that $\overline{\operatorname{conv}} S_{\Phi}=S \overline{\text { conv } \Phi} \Rightarrow S_{\text {cl } \Phi}=\overline{\operatorname{conv}} S_{\Phi}$.

Finally we can use $S_{\Phi}$ to extract information about $F(\cdot)$. Assume that $X$ is any separable Banach space.

PROPOSITION 3.2. $S_{\Phi}$ is convex if and only if $F(\cdot)$ is convex valued almost everywhere.

Proof. First assume that $S_{\Phi}$ is convex. Consider the operator 
$I: L_{X}^{1}(T) \rightarrow C(T ; X)$ defined by $(I f)(t)=\int_{0}^{t} f(s) d s$. This is clearly linear and $I\left(S_{F}^{1}\right)=S_{\Phi}$. Since convexity is preserved by the inverse image of a linear map we get that $S_{F}$ is convex. Then Corollary 1.6 of [16] tells us that $F(\cdot)$ is convex valued almost everywhere.

Finally if $F(\cdot)$ is convex valued almost everywhere, $S_{F}^{l}$ is convex and so $S_{\Phi}=I\left(S_{F}\right)$ is convex.

\section{Properties of the indefinite set valued integral}

We will start with a set valued version of a well-known result from integration theory. Assume that $X$ is any separable Banach space.

THEOREM 4.1. If $F: T \rightarrow P_{k c}(X)$ is integrably bounded then $\frac{1}{\varepsilon} \int_{t}^{t+\varepsilon} F(s) d s \stackrel{h}{\longrightarrow} F(t)$ almost everywhere as $\varepsilon \downarrow 0 \quad$ h is the Hausdorff metric).

Proof. Let $\psi_{n}(t)=\frac{1}{\varepsilon_{n}} \int_{t}^{t+\varepsilon}{ }^{n} F(s) d s$. We know that, for all $n \geq 1$, $t \rightarrow \psi_{n}(t), n \geq 1$, is measurable and closed, convex valued. Also for all $x^{*} \in X^{*}$ we have that $\sigma_{\psi_{n}(t)}\left(x^{*}\right)=\frac{1}{\varepsilon_{n}} \int_{t}^{t+\varepsilon} n \sigma_{F(s)}\left(x^{*}\right) d s$. Note that the dual unit ball $B^{*}$ with the weak* topology is compact and metrizable and $\sigma_{F(s)}\left(x^{*}\right)$ is continuous in $x^{*}$ on $B_{w^{*}}^{*}$ and measurable in $s$ and is bounded by $|F|(\cdot) \in L^{\perp}$. So invoking a result of Scorza-Dragoni [27] we deduce that $\lim _{n \rightarrow \infty} \frac{1}{\varepsilon_{n}} \int_{t}^{t+\varepsilon} n \sigma_{F(s)}\left(x^{*}\right) d s=\sigma_{F(t)}\left(x^{*}\right)$ for all $x^{*} \in X^{*}$ outside a null set independent of $x^{*}$ and the convergence is uniform on $B^{*}$. Since $\frac{1}{\varepsilon_{n}} \int_{t}^{t+\varepsilon_{n}} \sigma_{F(s)}\left(x^{*}\right) d s=\sigma \varepsilon_{n} \int_{t}^{t+\varepsilon_{F(s)}} n_{F}$ the theorem follows 
from Hörmander's formula.

REMARK. This result generalizes significantly Theorem 4.3 of Artstein [1] and Lemma 1.3 of Hermes [14].

In [3], Bradley and Datko introduced the following notion of differentiability of multifunctions.

DEFINITION 4.1 [3]. A multifunction $F: T \rightarrow P_{f c}(X)$ with bounded values is said to be differentiable at $t_{0} \in T$ if, for each $x^{*} \in X^{*}$ such that $\left\|x^{*}\right\|=1,(d / d t) \sigma_{F(t)}\left(x^{*}\right)$ exists at $t_{0}$ and equals $g\left(t_{0}, x^{*}\right)$ where $x^{*} \rightarrow g\left(t_{0}, x^{*}\right)$ is a continuous, positively homogeneous, sublinear function on $B^{*}$. In this case the derivative $D F\left(t_{0}\right)$ is the set $D F\left(t_{0}\right)=\bigcap_{x^{*} \in B^{*}}\left\{x \in X:\left(x^{*}, x\right) \leq g\left(t_{0}, x^{*}\right)\right\}$.

We will call this differentiability for multifunctions $D$ differentiability. Adopting the reasoning in the proof of Theorem 4.1 we can have the following result.

PROPOSITION 4.1. If $F: T \rightarrow P_{k c}(X)$ is integrably bounded then $D \Phi(t)=F(t)$ almost everywhere.

Next we will show that if the integrand multifunction has a nonempty interior, then so does the trajectory multifunction and the two are related in a natural way.

PROPOSITION 4.2. If $F: T \rightarrow P_{f e}(X)$ is integrably bounded and, for all $t \in T$, int $F(t) \neq \varnothing$ then, for all $t \in T$, int $\Phi(t)=\int_{0}^{t}$ int $F(s) d s$.

Proof. From Cronwall [7] we know that, for all $t \in T$, int $\Phi(t)=\operatorname{int} \int_{0}^{t} F(s) d s=\int_{0}^{t}$ int $F(s) d s$. It remains to show that $\int_{0}^{t}$ int $F(s) d s \neq \varnothing$. But recall that $\operatorname{Gr}($ int $F) \in \Sigma \times B(X)$. So we 
can apply Aumann's measurable selection theorem to find $f: T \rightarrow X$ such that $f(t) \epsilon$ int $F(t)$ for all $t \in T$. Clearly $f(\cdot) \in L_{X}^{l}(T)$. Hence $\int_{0}^{t}$ int $F(s) d s \neq \varnothing$.

The first part of the next theorem provides a complement to the previous result. It say that if at a certain time instant the trajectory multifunction has a nonempty interior, then this fact propagates into the future. The second part of the theorem gives us a necessary and sufficient condition for equality almost everywhere of two trajectories.

THEOREM 4.2. (i) If $F: T \rightarrow P_{w k c}(X)$ is integrably bounded and, for some $t \in T$, int $\Phi(t) \neq \varnothing$ then, for all $t^{\prime} \geq t$, int $\Phi\left(t^{\prime}\right) \neq \emptyset$.

(ii) If $F_{1}, F_{2}: T \rightarrow P_{w k c}(X)$ are integrably bounded then $\Phi_{1}(t)=\Phi_{2}(t)$ almost everywhere if and only if

$$
F_{1}(t)=F_{2}(t) \text { almost everywhere. }
$$

Proof. (i) Note that for $t^{\prime} \in T, t^{\prime}>t$, we have $\Phi\left(t^{\prime}\right)=\int_{0}^{t} F(s) d s=\int_{0}^{t} F(s) d s+\int_{t}^{t^{\prime}} F(s) d s=\Phi(t)+\int_{t}^{t^{\prime}} F(s) d s$.

So $\Phi\left(t^{\prime}\right) \supseteq \operatorname{int} \Phi(t)+\int_{t}^{t^{\prime}} F(s) d s$. Since the right hand side is nonempty and open we deduce that int $\Phi\left(t^{\prime}\right) \neq \varnothing$.

(ii) First suppose that cl $\Phi_{1}(t)=c l \Phi_{2}(t)$ almost everywhere. Then

$$
\sigma_{c l \Phi_{1}}(t)(\cdot)=\sigma_{\Phi_{1}}(t)(\cdot)=\sigma_{c l \Phi_{2}(t)}(\cdot)=\sigma_{\Phi_{2}}(t)(\cdot) \text { almost everywhere. }
$$

Recall that $\sigma_{\Phi_{1}(t)}(\cdot)=\int_{0}^{t} \sigma_{F_{1}}(s)(\cdot) d s$ and $\sigma_{\Phi_{2}}(t)(\cdot)=\int_{0}^{t} \sigma_{F_{2}}(s)(\cdot) d s$. Differentiating with respect to $t$ we have

$$
\frac{d}{d t} \int_{0}^{t} \sigma_{F_{1}}(s)(\cdot) d s=\sigma_{F_{1}}(t)(\cdot) \text { and } \frac{d}{d t} \int_{0}^{t} \sigma_{F_{2}(s)}(\cdot) d s=\sigma_{F_{2}}(t)(\cdot)
$$


almost everywhere and the exceptional null set is independent of the argument of the support function. Thus we have

$$
\begin{aligned}
\sigma_{F_{1}}(t) & (\cdot)=\sigma_{F_{2}(t)}(\cdot) \text { almost everywhere } \\
\Rightarrow F_{1}(t) & =F_{2}(t) \text { almost everywhere. }
\end{aligned}
$$

The converse is obvious.

REMARK. The sufficiency part is true if we assume instead that $r: T \rightarrow P_{f}(X)$ is integrably bounded and $\overline{\operatorname{conv}} F_{I}(t)=\overline{\operatorname{conv}} F_{2}(t)$ almost everywhere.

The last result of this section considers multifunctions paramatrized by elements in $X$ and examine their regularity properties.

THEOREM 4.3. If $F: T \times X \rightarrow P_{\text {wke }}(X)$ is a multifunction such that

(1) there exists a measurable multifunction $G: T \rightarrow P_{w k c}(X)$ such that $\sigma_{G(\cdot)}\left(x^{*}(\cdot)\right) \in L^{1}(T)$ for azz $x^{*}(\cdot) \in L_{X_{W^{*}}^{*}}^{\infty}(T)$ and $F(t, x) \subseteq G(t)$ for alz $x \in X$ almost everywhere;

(2) for all $t \in T, F(t, \cdot)$ is w-upper semi-continuous;

(3) for alz $x \in X, F(\cdot, \cdot)$ is measurable with $S_{F(\cdot, x)}^{1} \neq \emptyset$ and crosed in $L_{X}^{1}(\Omega)$.

Let $\Phi(t, x(\cdot))=\int_{0}^{t} F(s, x(\cdot)) d s$ where $x(\cdot) \in L_{X}^{1}(T)$. Then for all $t \in T,(t, \cdot)$ is w-upper semi-continuous on $L_{X}^{1}(T)$.

Proof. We will start the proof by showing that $\int_{0} F(s, x(s)) d s \neq \emptyset$. For $x^{*} \in X^{*}$ let $\varphi_{x^{*}}(t, x)=\sigma_{F(t, x)}\left(x^{*}\right)$. Note that, for all $x \in X$, $\varphi_{x^{*}}(t, x)$ is finite. Also from Theorem III-37 and II-20 of [6] we know respectively that $(t, x) \rightarrow \varphi_{x^{*}}(t, x)$ is measurable and $x \rightarrow \varphi_{x^{*}}(t, x)$ is upper semi-continuous. So $-\varphi_{x^{*}}(\cdot, \cdot)$ is normal in the sense of Rockafellar [24]. Because $\varphi_{x^{*}}(\cdot, \cdot)$ is finite for all $t$, we deduce 
that $\varphi_{x^{*}}(\cdot, \cdot)$ is $\sum \times B(X)$-measurable. So for any $x: T \rightarrow X$ measurable, we have that $t \rightarrow \varphi_{x^{*}}(t, x(t))$ is measurable. Once again invoke Theorem III-37 of [6] to deduce that $t \rightarrow F(t, x(t))$ is measurable. So we can find $f: T \rightarrow X$ measurable such that $f(t) \in E(t, x(t))$ for all $t \in T$. For all $x^{*} \in X^{*}$ we have that $\left(x^{*}, f(t)\right) \leq \sigma_{G(t)}\left(x^{*}\right)$ almost everywhere. Consider the multifunction

$$
H(t)=\left\{x^{*} \in B^{*}:\left(x^{*}, f(t)\right)=\|f(t)\|\right\} .
$$

From the Hahn-Banach theorem we know that, for all $t \in T, H(t) \neq \emptyset$. Also it is closed valued and it is easy to see that it is measurable. Hence we can find $x^{*}: T \rightarrow B^{*}$ strongly measurable such that $\left(x^{*}(t), f(t)\right)=\|f(t)\| \leq \sigma_{G(t)}\left(x^{*}(t)\right)$ almost everywhere, which implies that $f \in S_{F(\cdot, x(\cdot))}^{l} \neq \varnothing$. Clearly $S_{F(\cdot, x(\cdot))}^{l}$ is convex and as in the proof of Theorem 3.1 we can get that it is $w$-compact in $L_{X}^{1}(T)$. Now let $x_{n}(\cdot) \stackrel{L_{X}^{1}(T)}{\longrightarrow} x(\cdot)$. By passing to a subsequence we may assume that $x_{n}(t) \rightarrow x(t)$ almost everywhere. Then we have

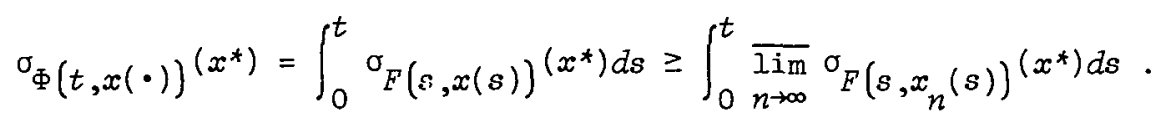

Applying Fatou's lemma we have finally that

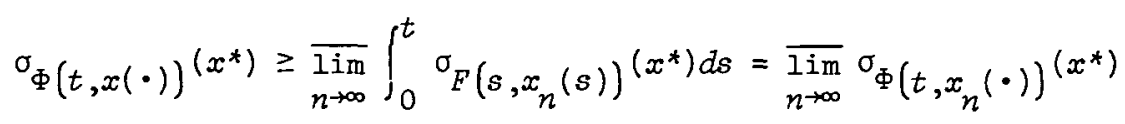

which proves that $x(\cdot) \rightarrow \sigma_{\Phi(t, x(\cdot))^{\left(x^{*}\right)}}$ is upper semi-continuous for the norm topology on $L_{X}^{1}(T)$ (or even the topology of convergence in measure). So finally Theorem II-20 of [6] tells us that $\Phi(t, \cdot)$ is weakly upper semi-continuous on $L_{X}^{1}(T)$.

\section{Convergence and stability results}

This section is devoted to the study of some convergence results in set valued integration and also includes an interesting stability result 
for $\Phi(\cdot)$ with potential applications in differential inclusions.

We start with a very general set valued version of the celebrated "dominated convergence theorem". Our theorem appears to be the most general one existing in the literature and improves significantly those obtained by Artstein [1] and Bridgland [5]. Assume $X$ is reflexive.

THEOREM 5.1. If $F_{n}, F: T \rightarrow P_{k c}(X)$ are multifunctions uniformly integrably bounded by $k(\cdot) \in L^{1}(T)$ and $F_{n}(t) \stackrel{K-M}{\longrightarrow} F(t)$ almost everywhere as $n \rightarrow \infty$ then

(i) $\Phi_{n}(t) \stackrel{K-M}{\longrightarrow} \Phi(t)$ almost everywhere as $n \rightarrow \infty$,

(ii) if $k(\cdot) \in L^{\infty}(T)$ then $S_{\Phi_{n}} \stackrel{K-M}{\longrightarrow} S_{\Phi}$ in $C(T ; X)$.

Proof. From Corollary 7A of Salinetti and Wets [25] we know that $\sigma_{F_{n}(s)}(\cdot) \rightarrow \sigma_{E(s)}(\cdot)$ almost everywhere as $n \rightarrow \infty$. Also we know that

$$
\sigma_{\Phi}(t)(\cdot)=\int_{0}^{t} \sigma_{F_{n}(s)}(\cdot) d s \text { and } \sigma_{\Phi(t)}(\cdot)=\int_{0}^{t} \sigma_{F(s)}(\cdot) d s \text {. }
$$

Applying Lebesgue's dominated convergence theorem we get that

$$
\lim _{n \rightarrow \infty} \sigma_{\Phi_{n}(t)}(\cdot)=\int_{0}^{t} \lim _{n \rightarrow \infty} \sigma_{F_{n}(s)}(\cdot) d s=\int_{0}^{t} \sigma_{F(s)}(\cdot) d s=\sigma_{\Phi(t)}(\cdot) .
$$

Since $\operatorname{dom} \sigma_{\Phi_{n}(t)}(\cdot)=\operatorname{dom} \sigma_{\Phi(t)}(\cdot)=X^{*}$ almost everywhere we can use Corollary 2E of [25] and get that $\sigma_{\Phi_{n}(t)}(\cdot) \stackrel{\tau^{*}}{\longrightarrow} \sigma_{\Phi(t)}(\cdot)$ almost everywhere as $n \rightarrow \infty$. But then Theorem 3.1 of Mosco [21] tells us that $\Phi_{n}(t) \stackrel{K-M}{\longrightarrow} \Phi(t)$ almost everywhere.

(ii) We will show that $w-\overline{\lim _{n \rightarrow \infty}} S_{\Phi_{n}} \stackrel{\check{\subseteq}}{\subseteq} S_{\Phi} \subseteq s-\frac{\lim }{n \rightarrow \infty} S_{\Phi_{n}}$. To this end let $\varphi \in w-\overline{\lim }_{n \rightarrow \infty} S_{\Phi_{n}}$. By definition we know that there exists a subsequence $\{m\} \subseteq\{n\}$ such that $\varphi_{m} \in S_{\Phi_{n}}$ and $\varphi_{m} \stackrel{w-C(T ; X)}{\longrightarrow} \varphi$ as $m \rightarrow \infty$. 
For all $m \geq 1$ we have $\varphi_{m}(t)=\int_{0}^{t} f_{m}(s) d s$ where $f_{m} \in s_{F_{m}}^{1}$. From Alaoglu's theorem $B_{\|k\|_{\infty}}(0)=\left\{x \in X:\|x\| \leq\|k\|_{\infty}\right\}$ is w-compact and, for all $m \geq 1, f_{m}(\omega) \in B_{\|k\|_{\infty}}(0)$ almost everywhere. Hence Theorem 2 of Diestel [11] tells us that $\left\{f_{m}\right\}_{m \geq 1}$ is relatively $w$-compact in $L_{X}^{l}(\Omega)$. So we can find a further subsequence $\{l\} \subseteq\{m\}$ such that $f_{Z} \stackrel{w-L_{X}^{1}(T)}{\longrightarrow} f$. Our claim is that $f \in S_{F}$. If not there exists $\varepsilon>0$ and $g(\cdot) \in L_{X^{*}}^{\infty}(T)$ such that $\langle g, f\rangle-\varepsilon \geq \underset{S_{F}}{\sigma}(g)$ where $\langle\cdot, \cdot\rangle$ are the duality brackets for the dual system $\left(L_{X}^{1}(T), L_{X^{*}}^{\infty}(T)\right)$. Recall that $\sigma_{S_{F}^{1}}(g)=\int_{0}^{T} \sigma_{F(s)}(g(s)) d s$. Also $\int_{0}^{T} \sigma_{F_{Z}(s)}(g(s)) d s \rightarrow \int_{0}^{T} \sigma_{F(s)}(g(s)) d s$. So for $\tau$ sufficiently large we have that

$$
\left\langle g, f_{Z}\right\rangle-\frac{\varepsilon}{3} \geq \int_{0}^{T} \sigma_{F_{Z}(s)}(g(s)) d s=\sigma_{s_{E_{Z}}^{l}}(g)
$$

a contradiction, because $f_{l} \in S_{F_{l}}^{1}$. Thus $f \in S_{F}^{l}$. From Theorem 3 (p. 387) of Dinculeanu [11] we know that $[C(T ; X)]^{*}=M_{p}\left(\Sigma ; X^{*}\right)$. So for $n(\cdot) \in M_{r}\left(\Sigma ; X^{*}\right)$ we have that

$$
\left\langle\varphi_{Z}, m\right\rangle=\int_{0}^{T} \varphi_{Z}(s) d m(s) \rightarrow \int_{0}^{T} \varphi(s) d m(s)=\langle\varphi, m\rangle
$$

where $(\cdot, \cdot)$ are now the duality brackets for $\left(C(T ; X), M_{p}\left(\Sigma ; X^{*}\right)\right)$. Imploying Lebesgue's dominated convergence theorem we can write that

$$
\lim _{l \rightarrow \infty} \int_{0}^{t} \int_{0}^{t} f_{\zeta}(s) d s d m(t)=\int_{0}^{T} \lim _{l \rightarrow \infty} \int_{0}^{t} f_{\zeta}(s) d s d m(t)=\int_{0}^{T} \int_{0}^{t} f(s) d s d m(t) .
$$

Since $m(\cdot) \in M_{p}\left(\Sigma ; X^{*}\right)$ was arbitrary we deduce that 
$\varphi(t)=\int_{0}^{t} f(s) d s$. So $\varphi \in S_{\Phi}$ which means that

$$
w-\varlimsup_{n \rightarrow \infty} S_{\Phi_{n}} \subseteq S_{\Phi}
$$

Next let $\varphi \in S_{\Phi}$. Then there exists $f \in S_{F}^{1}$ such that $\varphi(t)=\int_{0}^{t} f(s) d s$. Define $L_{n}(s)=\left\{\hat{x} \in X:\|f(x)-\hat{x}\|=\inf _{x \in F}(s)\|f(s)-x\|\right\}$, $n \geq 1$. Because $F_{n}(s) \in P_{w k e}(X), s \in T, n \geq 1$, and $x \rightarrow\|f(s)-x\|$ is lower semi-continuous, from Weierstrass' theorem we get that $L_{n}(s)$ is nonempty and closed for all $s \in T$ and all $n \geq 1$. Also Lemma 2.I of [16] tells us that $s \rightarrow \underset{\text { inf }}{\|f(s)-x\|}$ is measurable and this in turn $x \in F_{n}(s)$

implies that $s \rightarrow L_{n}(s)$ is measurable. So we can find $f_{n}: T \rightarrow X$

measurable such that $f_{n}(s) \in L_{n}(s), s \in T, n \geq 1$. Hence $\left\|f(s)-F_{n}(s)\right\|=\left\|f(s)-f_{n}(s)\right\| \rightarrow 0$ as $n \rightarrow \infty$. Thus

$$
\varphi_{n}(t)=\int_{0}^{t} f_{n}(s) d s \rightarrow \varphi(t)=\int_{0}^{t} f(s) d s
$$

as $n+\infty$. Consider

$$
\begin{aligned}
\left\|\varphi_{n}-\varphi\right\|_{\infty} & =\sup _{t \in T}\left\|\varphi_{n}(t)-\varphi(t)\right\|=\sup _{t \in T}\left\|\int_{0}^{t}\left(f_{n}(s)-f(s)\right) d s\right\| \\
& \leq \sup _{t \in T} \int_{0}^{t}\left\|f_{n}(s)-f(s)\right\| d s \\
& =\left\|f_{n}-f\right\|_{L_{X}^{l}(T)} \rightarrow 0 \text { as } n \rightarrow \infty .
\end{aligned}
$$

So we have that $\varphi_{n} \stackrel{C(T ; X)}{\longrightarrow} \varphi$ as $n \rightarrow \infty$. This then means that $\varphi \in s-\frac{\lim }{n \rightarrow \infty} S_{\Phi_{n}}$ which in turn implies that

$$
S_{\Phi} \subseteq s-\underline{\lim } S_{\Phi_{n}}
$$

From (1) and (2) above we finally conclude that 


$$
S_{\Phi_{n}} \stackrel{K-M}{\longrightarrow} S_{\Phi}
$$

as $n \rightarrow \infty$.

Next we pass to multivalued generalizations of Fatou's lemma.

THEOREM 5.2. If $F_{n}: T \rightarrow P_{w k c}(X), n \geq 1$, are uniformly integrably bounded by $g(\cdot) \in L^{1}(\Omega)$ and, for all $t \in T$,

$$
s-\frac{\lim }{n \rightarrow \infty} F_{n}(t) \in P_{w k c}(X)
$$

then

$$
\int_{0}^{t} s-\frac{\lim }{n \rightarrow \infty} E_{n}(s) d s \subseteq s-\frac{\lim }{n \rightarrow \infty} \int_{0}^{t} F_{n}(s) d s
$$

Proof. If $s-\frac{\lim }{n \rightarrow \infty} F_{n}(t)=\emptyset$ almost everywhere then the conclusion of the theorem is obvious. So assume without loss of generality that $s-\frac{\lim }{n \rightarrow \infty} F_{n}(t) \neq \emptyset$ for all $t \in T$. Since $s-\frac{\lim }{n \rightarrow \infty} F_{n}(t) \in P_{w k c}(X)$ and since $t \rightarrow s-\frac{\lim }{n \rightarrow \infty} F_{n}(t)$ is measurable (see [26]), $S_{\Phi} \neq \emptyset$ where $\tilde{\Phi}(t)=\int_{0}^{t} s-\frac{\lim }{n \rightarrow \infty} F_{n}(s) d s$. Let $\varphi \in S_{\Phi}$ and $\Phi_{n}(t)=\int_{0}^{t} F_{n}(s) d s$. Consider $\Gamma_{n}(t)=\left\{x \in \Phi_{n}(t):\|\varphi(t)-x\|=\left\|\varphi(t)-\Phi_{n}(t)\right\|\right\}$. Recalling that $\tilde{\Phi}_{n}(t) \in P_{w k e}(X), t \in T, n \geq 1$, we can show as before that $\Gamma_{n}: T \rightarrow P_{\omega k c}(X)$ and is measurable. So we can find $\varphi_{n}: T+X$ measurable such that $\varphi_{n}(t) \in \Gamma_{n}(t), t \in T, n \geq 1$. Then we have 


$$
\begin{aligned}
& \left\|\varphi(t)-\varphi_{n}(t)\right\|=\left\|\varphi(t)-\Phi_{n}(t)\right\|=\inf _{f_{n} \in S_{F_{n}}^{1}}\left\|\varphi(t)-\int_{0}^{t} f_{n}(s) d s\right\| \\
& =\inf _{f_{n} \in S_{F_{n}}^{1}}\left\|\int_{0}^{t}\left(f(s)-f_{n}(s)\right) d s\right\|\left(f \in S_{s-\frac{1 i m}{n \rightarrow \infty} F_{n}}^{l}\right) \\
& \leq \inf _{f_{n} \in S_{F_{n}}^{1}} \int_{0}^{t}\left\|f(s)-f_{n}(s)\right\| d s \\
& =\int_{0}^{t} \inf _{x \in F_{n}(s)}\|f(s)-x\| d s \\
& =\int_{0}^{t}\left\|f(s)-F_{n}(s)\right\| d s \text {. }
\end{aligned}
$$

Since $f(s) \in s-\frac{\lim _{n \rightarrow \infty}}{F_{n}}(s)$ set, we know that $\left\|f(s)-F_{n}(s)\right\| \rightarrow 0$ as $n \rightarrow \infty$. So $\int_{0}^{t}\left\|f(s)-F_{n}(s)\right\| d s \rightarrow 0$ as $n \rightarrow \infty \Rightarrow\left\|\varphi(t)-\varphi_{n}(t)\right\| \rightarrow 0$ as $n \rightarrow \infty$. Thus $\varphi(t) \in s-\frac{\lim }{n \rightarrow \infty} \Phi_{n}(t)$ for all $t \in T$. From this we conclude that $\int_{0}^{t} s-\frac{\lim }{n \rightarrow \infty} F_{n}(s) d s \subseteq s-\frac{\lim }{n \rightarrow \infty} \int_{0}^{t} F_{n}(s) d s$ as desired.

We have a version of this theorem involving limits superior.

THEOREM 5.3. If $F_{n}: T \rightarrow P_{w k c}(X), n \geq 1$, are measurable multifunctions such that $s-\overline{\lim }_{n \rightarrow \infty} F_{n}(t) \neq \varnothing$ for all $t \in T$ and, for all $n \geq 1, S_{F_{n}}^{1} \subseteq W$ where $W$ is weakly compact in $L_{X}^{\infty}(T)$ then $s-\overline{\lim }_{n \rightarrow \infty}^{t} \int_{0} F_{n}(s) d s \subseteq \int_{0}^{t} w-\overline{\lim }_{n \rightarrow \infty} F_{n}(s) d s$.

Proof. We know that for all $n \geq 1$ and all $t \in T, \Phi_{n}(t) \in P_{w k c}(X)$ and that $t \rightarrow s-\varlimsup_{n \rightarrow \infty} \Phi_{n}(t)$ is measurable. Let $\varphi \in S_{s-\overline{\lim }_{n \rightarrow \infty} \Phi_{n}}$. Then for all $t \in T, \varphi(t) \in s-\overline{\lim _{n \rightarrow \infty}} \Phi_{n}(t)$. As before we can find $\varphi_{n} \in S_{\Phi_{n}}$ such 
that $\left\|\varphi(t)-\varphi_{n}(t)\right\|=\left\|\varphi(t)-\Phi_{n}(t)\right\|$ for all $t \in T, n \geq 1$. Recall that $\underset{n \rightarrow \infty}{\lim }\left\|\varphi(t)-\Phi_{n}(t)\right\|=0$. Let $f_{n} \in S_{F_{n}}^{l}$ such that $\varphi_{n}(t)=\int_{0}^{t} f_{n}(s) d s$. Because $\left\{f_{n}\right\}_{n \geq 1} \subseteq W$ we can find a subsequence $\left\{f_{n_{k}}\right\}_{k \geq 1} \subseteq\left\{f_{n}\right\}_{n \geq 1}$ such that $f_{n_{k}} \stackrel{w-L_{X}^{\infty}(T)}{\longrightarrow} f$ as $k \rightarrow \infty$. From Khurana [20] we get that $f_{n_{k}}(t) \stackrel{w}{\longrightarrow} f(t)$ almost everywhere which means that $f \in S_{w-\overline{\lim }_{n \rightarrow \infty} F_{n}} \cdot$ Also we have that, for all $t \in T, \int_{0}^{t} f_{n_{k}}(s) d s \stackrel{\omega}{\longrightarrow} \int_{0}^{t} f(s) d s$. Exploiting the well-known fact that the norm functional is $w$ lower semi-continuous, we deduce that

$$
\begin{aligned}
& 0=\frac{\lim }{n \rightarrow \infty}\left\|\varphi(t)-\int_{0}^{t} f_{n_{k}}(s) d s\right\| \geq\left\|\varphi(t)-\int_{0}^{t} f(s) d s\right\| \Rightarrow\left\|\varphi(t)-\int_{0}^{t} f(s) d s\right\|=0 \\
& \Rightarrow \varphi(t)=\int_{0}^{t} f(s) d s \text {. }
\end{aligned}
$$

Thus $\varphi(t) \in \int_{0}^{t} w-\overline{\lim } F(s) d s$ from which we conclude that $s-\varlimsup_{n \rightarrow \infty} \int_{0}^{t} F_{n}(s) d s \subseteq \int_{0}^{t} w-\varlimsup_{n \rightarrow \infty} F_{n}(s) d s$.

REMARK. If $X$ is finite dimensional, then the weak and strong topologies coincide and so we can write $\varlimsup_{n \rightarrow \infty} \int_{0}^{t} F_{n}(s) d s \subseteq \int_{0}^{t} \overline{\lim _{n \rightarrow \infty}} F_{n}(s) d s$. It will be very interesting, especially for mathematical economics (core equivalence theory) to have a version of the above theorem without the condition on the sets $S_{F_{n}}$ and have both limits superior taken in the same topology .

We will conclude our work with a result that is important in the theorem of differential inclusions and in particular in problems dealing with the peripheral attainability of state. 
THEOREM 5.4. If $F: T \rightarrow P_{f c}(X)$ is measurabie, $S_{E}^{1} \neq \emptyset$ and int $E(t) \neq \varnothing$ almost everywhere then $\varphi(t) \in b d \Phi(t)$ for all $t \in T$ implies that $\dot{\varphi}(t) \in b d F(t)$ almost everywhere.

Proof. Suppose not. Then we can find $A \in \Sigma$ with $\lambda(A)>0$ such that $\dot{\varphi}(t) \notin b d F(t)$ for all $t \in A$. On this set consider the multifunction

$$
\begin{aligned}
t \rightarrow \Gamma(t) & =\left\{\varepsilon>0: B_{\varepsilon}(\dot{\varphi}(t)) \subseteq F(t)\right\} \\
& =\{\varepsilon>0:\|\dot{\varphi}(t)-b d F(t)\| \geq \varepsilon\} .
\end{aligned}
$$

So $\operatorname{Gr} \Gamma=\left\{(t, \varepsilon) \in A \times\left(\mathbf{R}_{+} \backslash\{0\}\right): \dot{\varphi}(t)-b d F(t)-\varepsilon \geq 0\right\}$. Let $u(t, \varepsilon)=\|\dot{\varphi}(t)-b d F(t)\|-\varepsilon$. From Theorem 4.6 of Himmelberg [18] we know that $t \rightarrow b d F(t)$ is a measurable multifunction. So $t \rightarrow\|\dot{\varphi}(t)-b d F(t)\|$ is measurable (see Theorem 2.1). Hence $u(\cdot, \cdot)$ is a Caratheodory function and so is jointly measurable. This then implies that $\operatorname{Gr} \Gamma \in \Sigma_{A} \cap B\left(\mathrm{R}_{+}\right)$ where $\Sigma_{A}=\Sigma \cap A$. Applying Aumann's selection theorem we can find $\varepsilon: A \rightarrow R_{+} \backslash\{0\}$ measurable such that $\varepsilon(t) \in \Gamma(t)$ for all $t \in A$. Then we have that $\dot{\varphi}(t)+B_{\varepsilon(t)}(\dot{\varphi}(t)) \subseteq F(t)$ for all $t \in A$. Let

$$
G(t)=\left\{\begin{array}{l}
B_{\varepsilon(t)}(\dot{\varphi}(t)), t \in A, \\
\{0\}, t \in T \backslash A .
\end{array}\right.
$$

Clearly $G(\cdot)$ is closed valued, measurable and $S_{G}^{l} \neq \emptyset$. We have $\dot{\varphi}(t)+G(t) \subseteq F(t)$ for all $t \in T$ implies

$$
\begin{aligned}
\int_{0}^{t} \dot{\varphi}(s) d s+\int_{0}^{t} G(s) d s \subseteq \int_{0}^{t} E(s) d s=\Phi(t) & \\
\Rightarrow \varphi(t)-\varphi(0)+\int_{A \cap[0, t]}{ }^{B} \varepsilon(s) & (\dot{\varphi}(s)) d s \subseteq \Phi(t) \\
& \Rightarrow \varphi(t)+\int_{A \cap[0, t]}{ }^{B}{ }_{\varepsilon(s)}(\dot{\varphi}(s)) d s \subseteq \Phi(t) .
\end{aligned}
$$

But from Cornwall [7] we know that 


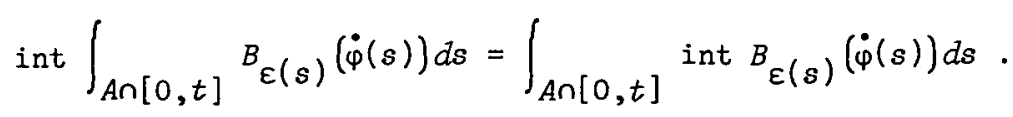

Therefore we conclude that $\varphi(t) \in$ int $\Phi(t)$ for all $t \in T$ a contradiction.

More results in this direction were recently obtained by the author in [22]. One of them is a general differentiability result for multifunctions that generalizes significantly Theorem 4.1 of Artstein [1] and also introduces a different technique which is more appropriate when dealing with multifunctions with values in an infinite dimensional Banach space. This approach is based on the theory of multimeasures and vector measures. For details and other representation results we refer to [22].

\section{References}

[1] 2. Artstein, "On the calculus of closed set valued functions", Indiana Univ. Math. J. 24 (1974), 433-441.

[2] Robert J. Aumann, "Integrals of set-valued functions", J. Amth. Anal. App 2. 12 (1965), 1-12.

[3] M. Bradley and R. Datko, "Some analytic and measure theoretic properties of set valued mappings", SIAM J. Control Optim. 15 (1977), 625-635.

[4] T.F. Bridgland, Jr., "Trajectory integrals of set valued functions", Pacific J. Math. 33 (1970), 43-68.

[5] J.F. Bridgland, Jr., "Extreme limits of compacta valued functions", Trans. Amer. Math. Soc. 170 (1972), 149-163.

[6] Ch. Castaing and M. Valadier, Convex analysis and measurable multifunctions (Lecture Notes in Mathematics, 580. Springer-Verlag, Berlin, Heidelberg, New York, 1977).

[7] R. Cornwall, "Conditions for the graph and the integral of a correspondence to be open", J. Math. Anal. Appl. 39 (1972), $771-792$.

[8] R. Curtain and A. Pritchard, Eunctional analysis in modern applied mathematics (Academic Press, New York, London, 1977). 
[9] J. Diestel, "Remarks on weak compactness in $L^{\perp}(U ; X)$ ", Glasgow Math. J. 18 (1977), 87-91.

[10] J. Diestel and J.J. UhI, Vector measures (Mathematical Surveys, 15. American Mathematical Society, Providence, Rhode Island, 1977).

[11] N. Dinculeanu, Vector measures (Pergamon Press, London, 1967).

[12] Nelson Dunford and Jacob T. Schwartz, Linear operators. I (Interscience [John Wiley \& Sons], New York, London, 1958).

[13] K. Floret, Weakly compact sets (Lectures Notes in Mathematics, 801. Springer-Verlag, Berlin, Heidelberg, New York, 1980).

[14] H. Hermes, "Calculus of set valued functions", J. Math. Mech. 18 (1968), 47-60.

[15] H. Hermes, "The generalized differential equation $x \in R(t, x)$ ", Adv. in Math. 4 (1970), 149-169.

[16] F. Hiai and H. Umegaki, "Integrals, conditional expectations and martingales of multivalued functions", J. MuZtivariate Anal. 7 (1977), 149-182.

[17] W. Hildenbrandt, Core and equilibria of large economies (Princeton University Press, Princeton, New Jersey, 1974).

[18] C.J. Himmelberg, "Measurable relations", Fund. Math. 87 (1975), $53-72$.

[19] C.J. Himmelberg, T. Parthasarathy and F.S. van Vieck, "Un measurable relations", Fund. Math. 111 (1981), 161-167.

[20] S. Khurana, "Weak sequential convergence in $L_{E}^{\infty}$ and Dunford-Pettis property of $L_{E}^{1}$ ", Proc. Amer. Math. Soc. 78 (1980), 85-88.

[21] U. Mosco, "On the continuity of the Young-Funchel transform", $J$. Math. Anal. Appl. 35 (1971), 518-535.

[22] Nikolaos S. Papageorgiou, "Representation of set valued operators", Trans. Amer. Math. Soc. (to appear).

[23] R.T. Rockafellar, "Measurable dependence of convex sets and functions in parameters", J. Math. AnaZ. Appl. 28 (1969), 4-25. 
[24] R.T. Rockafellar, "Integral functionals, normal integrands and measurable selectors", Nonlinear operators and calculus of variations, 156-209 (Lecture Notes in Mathematics, 543. Springer-Verlag, Berlin, Heidelberg, New York, 1976).

[25] G. Salinetti and R. Wets, "On the relations between two types of convergence for convex functionals", J. Math. Anal. Appl. 60 (1977), 211-226.

[26] G. Salinetti and R. Wets, "On the convergence of closed valued measurable multi-functions", Trans. Amer. Math. Soc. 266 (1981), $274-289$.

[27] G. Scorza-Dragoni, "Sulla derivazione degli integrali indefinita", Rend. Acad. Naz. Lincei. 20 (1956), 711-714.

Added in Proof. Concerning the remark of p. 407 the author has recently proved a new Fatou's Lemma for $w-\overline{l i m}$ in $L_{X}^{1}$. This result appears in "On set valued random variables taking values in a separable Banach space", submitted.

Department of Mathematics, University of IIIinois, 1409 West Green Street, Urbana, IIIinois 61801 , USA. 